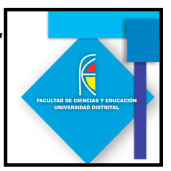

\title{
CONSTRUCCIÓN DE UNA MÁQUINA DE GOLDBERG PARA POTENCIAR LA OBSERVACIÓN Y LA DESCRIPCIÓN DE ESTUDIANTES DE GRADO SEXTO
}

\section{CONSTRUCTION A GOLDBERG MACHINE TO ENHANCE THE OBSERVATION AND DESCRIPTION OF SIXTH GRADE STUDENTS}

\begin{abstract}
Resumen
La máquina de Rude Goldberg, es un aparato que consiste en una sucesión de movimientos sencillos o complejos con un objetivo final, aunque parece tener como función el entretenimiento de quien la observa y diseña, puede ser utilizada en el aula de clase para la enseñanza de la física.

De esta manera y desde el espacio propiciado en uno de los seminarios de la Licenciatura en Física de la Universidad Pedagógica Nacional, llamado Construcción de Material de Laboratorio, se elabora una máquina de Goldberg, cuyo objetivo principal es potenciar las habilidades de observación y descripción de los estudiantes del grado sexto del Colegio Nuestra Señora de la Salette, con la apreciación de los diferentes tipos de movimiento presentes en la máquina y que se estudiaron con anterioridad en la clase de física.
\end{abstract}

Palabras Clave: Máquina de Goldberg, observación, descripción, movimiento.

\section{Abstract

\footnotetext{
${ }_{1}^{1}$ Universidad Pedagógica Nacional, dfi608_ygualtero@pedagogica.edu.co,

${ }^{2}$ Universidad Pedagógica Nacional, dfi443_jpatino@pedagogica.edu.co

${ }^{3}$ Universidad Pedagógica Nacional, dfi632_yrivera@pedagogica.edu.co

${ }^{4}$ Universidad Pedagógica Nacional, dfi463_agomez@pedagogica.edu.co
}

Rude Goldberg machine is a device that consists of sequence of simple or complex movements with an ultimate goal, but appears to have the entertainment function of the beholder and designs, can be used in the classroom for teaching physics. 


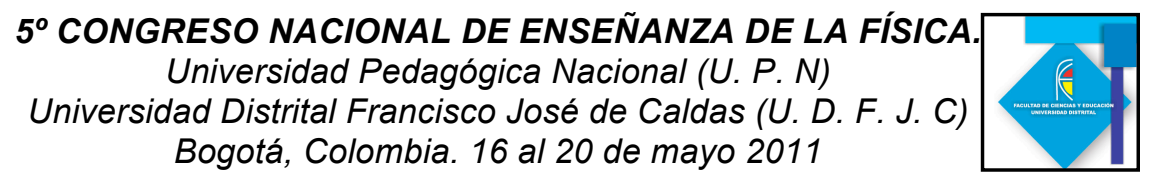

In this way and space resulted in one of the seminars for the Physics Degree in the Universidad Pedagógica Nacional named Material laboratory Construction, it develops Goldberg Machine, whose main objective to enhance the skills of observation and description sixth grade students in the College Nuestra Señora de la Salette, the assessment of different types of movement on the machine and previously studied in physics class.

Key words: Goldberg Machine, observation, description, motion.

\section{Introducción}

\section{Planteamiento del proyecto}

El Ministerio de Educación Nacional en los lineamientos curriculares para el área de ciencias, plantea que

"Una de las metas fundamentales de la formación en ciencias es procurar que los y las estudiantes se aproximen progresivamente al conocimiento científico, tomando como punto de partida su conocimiento "natural" del mundo."(MEN, 1996).

Desde esta perspectiva, las concepciones que tiene el ser humano sobre el mundo que lo rodea las obtiene por medio de la observación, comprendiendo que este proceso puede realizarse haciendo uso exclusivo de los sentidos o utilizando herramientas que el mismo hombre ha diseñado, como las escalas e instrumentos de medición. Sin embargo, más allá de la observación que se realice de un fenómeno, es muy importante la forma de comunicar lo que se observa en términos propios de la física.

Se considera que si un estudiante puede realizar a cabalidad el anterior proceso, se da un primer paso hacia la constitución de un ciudadano científicamente culto, capaz de comprender el mundo que lo rodea, tal como lo planteó Crespo (2003). Con lo mencionado se buscó que los estudiantes de grado sexto potencializaran la observación de fenómenos, brindándoles herramientas para que organizaran sus observaciones y comunicaran el resultado de las mismas a través de la descripción.

Se escogió esta población en particular por la curiosidad natural que presentan ante los fenómenos presentes en la cotidianidad, planteando como pregunta: ¿qué tipo de experiencia puede llamar la atención del estudiante para que se interese en observarla y describirla?

De esta manera y desde el espacio propiciado en el seminario de Construcción de Material de Laboratorio, se planteó construir una Máquina de Goldberg con materiales económicos, donde los estudiantes de grado sexto pudieran apreciar los distintos tipos de movimientos correspondientes a la cinemática que trabajaron en la clase de física.

\section{Construcción del Material}




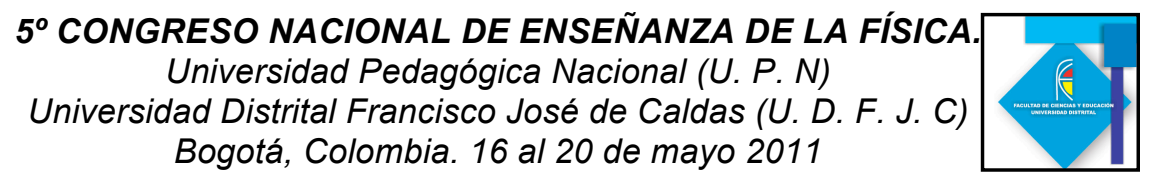

Se realizó una búsqueda que orientara la construcción del artefacto, donde se tuvieron en cuenta algunas consideraciones físicas para su elaboración. Se hicieron varios diseños pensando en el grupo donde se quería implementar la propuesta y se concretó una máquina en la que se evidenciara el movimiento uniforme, no uniforme, rectilíneo, curvilíneo y circular (Figura 1).

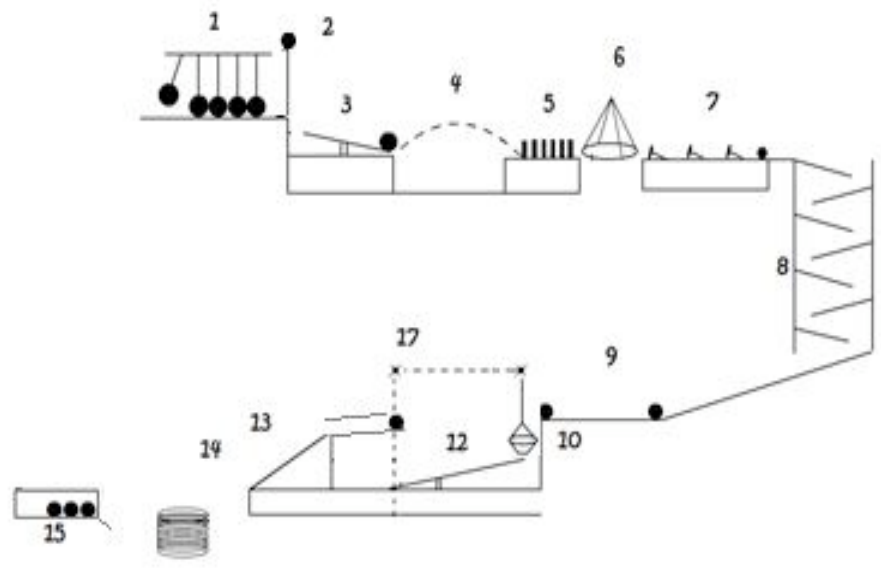

Figura 1

Con el diseño ya listo se empezaron a construir cada uno de los elementos, teniendo en cuenta que la máquina tendría una dimensión de $1 \mathrm{~m}^{2}$; como el material debía ser lo suficientemente robusto como para trabajarse varias veces, pero al mismo tiempo flexible para poder diseñar cada una de las etapas, se decidió trabajar las bases con madera y los elementos con cartón paja y balso, materiales que son de fácil adquisición.

Al tener construida cada una de las etapas de la máquina se empezó la fase de acoplamiento y ensamble, para lo cual fue necesario realizar repetidos ensayos para ajustar las posiciones exactas donde funcionaba a cabalidad este aparato. Al tener listo el esqueleto de la máquina se mejoró su apariencia para llamar la atención de los estudiantes.

\section{Descripción del Montaje Experimental}

Cada una de las etapas de la máquina se caracterizó por tener un determinado movimiento que fue posible observar en el funcionamiento de la misma. El primer proceso que se observó fue la Cuna de Newton, cinco esferas inelásticas suspendidas en fila y alineadas por sus centros, se desplazó la primera de su posición de equilibrio donde el centro de masa de la esfera ascendió una altura h, al caer se evidenció una conservación de la cantidad de movimiento a través de cada una de las esferas, de tal forma que la última golpeó un soporte vertical que sostenía en el borde superior una ficha de dominó, ésta cayó describiendo una caída libre.

En la tercera parte, la ficha de dominó que cayó libremente golpeó un extremo de un sube y baja, ocasionando que una esfera ubicada en el otro extremo realizara un movimiento semi-parabólico que terminaba en una barrera de cartón. 


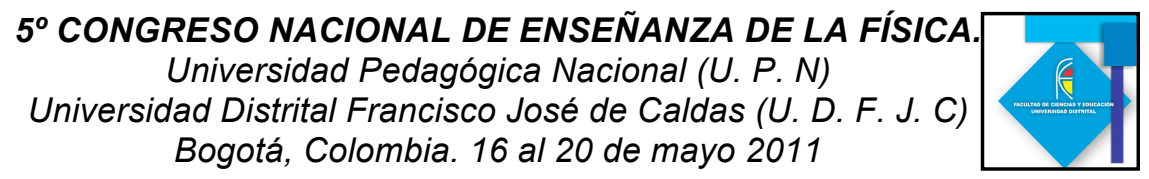

Cuando la esfera chocó con la barrera, ésta cayó y desencadenó un efecto dominó que finalmente hizo girar un disco suspendido; el disco describió un movimiento circular uniforme y golpeó una puntilla cabezona que a la vez transmitió su movimiento a dos más. La última puntilla golpeó a una esfera y ésta cayó por una serie de planos inclinados que terminaban con un movimiento rectilíneo.

La esfera terminó su movimiento rectilíneo cuando cayó en una canasta que golpeó un extremo de un sube y baja, que a su vez golpeó la parte baja de una manguera que sostenía una esfera, lo cual ocasionó que la esfera se deslizara a través ésta, hasta caer en una superficie elástica. Debido al choque que se produjo, la esfera abrió la puerta de una caja llena de canicas en su interior, cumpliendo así con el objetivo final de la máquina de Goldberg.

\section{Implementación}

El objetivo de la implementación era poder utilizar la máquina como un recurso para lograr que los estudiantes potencializaran la observación y realizaran mejores descripciones utilizando conceptos propios de la física. Para esto, se construyó un documento orientador que junto con el material de laboratorio elaborado, se implementó en cuatro etapas en el grado sexto del Colegio Nuestra Señora de la Salette. Los nombres y características de cada una de las etapas que estuvieron explicitas en el documento orientador fueron:

1. Acercamiento: Observación del funcionamiento de la Máquina de Goldberg y primera descripción.

2. Para recordar: Socialización de los conceptos trabajados con anterioridad en la clase de física.

3. Usando tus Recuerdos: Nueva observación de la Máquina de Goldberg en funcionamiento y organización de la información.

4. Mi nueva forma de ver las cosas: Caracterización de los fenómenos observados en el montaje experimental, teniendo en cuenta el lenguaje propio de la física, involucrando conceptos de trayectoria, distancia, velocidad, rapidez, aceleración y tipos de movimiento.

\section{Desarrollo y Resultados de la Implementación}

En la primera sesión, se llevan a cabo las dos primeras etapas (Acercamiento - Para recordar), se entregaron los documentos orientadores a los estudiantes para que ellos realizaran una primera lectura, uno de los maestros realizó una explicación para aclarar las dudas que los estudiantes presentaron.

Una vez esta orientación se hizo explícita en la clase, se puso la máquina en funcionamiento y se pidió a los estudiantes que realizaran la respectiva descripción de ésta. Sin embargo en los escritos que presentaron los estudiantes fue posible evidenciar la falta del lenguaje propio de la física, por lo que se propuso una discusión alrededor de cinco términos (trayectoria, desplazamiento, rapidez, velocidad y aceleración), con los cuales se puede hacer una caracterización del movimiento. Esta actividad permitió que los 


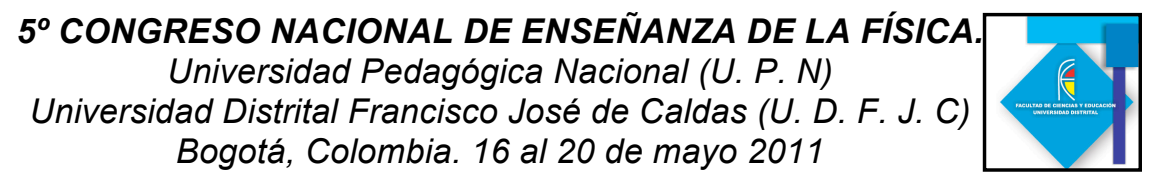

estudiantes recordaran el significado de estos conceptos de sus clases anteriores, además permitió la relación de los mismos, con fenómenos presentes en la vida cotidiana.

En la segunda sesión se retomó la socialización realizada en la clase anterior y se le pidió a los estudiantes que por grupos de cuatro personas le dieran un nombre a la máquina de Goldberg e hicieran una nueva descripción de ésta, pero esta vez, empleando un cuadro (diseñado por ellos mismos) donde fuera posible caracterizar físicamente cada uno de los pasos.

Con esta clasificación realizada por los estudiantes, fue posible evidenciar cómo con unos parámetros establecidos, ellos pudieron hacer descripciones detalladas de las características físicas que distinguían a cada uno de los pasos de la máquina de Goldberg, propiciando que describieran el montaje teniendo propiedad de los conceptos que dan cuenta del movimiento.

Concluyendo la segunda sesión, se dio inicio a la socialización de los cuadros que cada uno de los grupos planteó y el maestro propuso la actividad en casa que constaba de una presentación creativa del funcionamiento de la máquina hecha por cada uno de los estudiantes.

\section{Conclusiones}

Con la implementación de una ruta de trabajo para la enseñanza del movimiento y su relación con fenómenos cotidianos, fue posible lograr un aprendizaje significativo de conceptos.

El diseño y la implementación de documentos orientadores para el aprendizaje del movimiento y su relación con fenómenos específicos (máquina de Goldberg), evidenciaron una serie de constructos por parte de los estudiantes que fueron evolucionando a medida que se desarrollaban las sesiones de trabajo.

El trabajo que se llevó a cabo por grupos, permitió confrontar los conceptos y definiciones que los estudiantes tenían alrededor del tema de movimiento.

Con el trabajo en el aula fue posible ver que los estudiantes pasaron de hacer descripciones simples del funcionamiento de la máquina de Goldberg a utilizar el lenguaje científico para describir los movimientos presentes en ésta.

La máquina de Goldberg potencializó los procesos de observación del estudiante, dando al docente una herramienta didáctica e interactiva para llevar al aula.

\section{Referencias Bibliográficas}

Crespo, M. A. (2003). Concepciones del aprendizaje y la enseñanza: las nuevas propuestas educativas. Enseñar Ciencias en Secundaria. Concepciones del aprendizaje y la enseñanza: las nuevas propuestas educativas, (pág. 16). Bilbao.

MEN, M. d. (1996). Estándares básicos en competencias. 